\title{
Perrier : la mise en système médiatique d'une crise
}

\section{Violaine Appel}

\section{(2) OpenEdition}

Journals

Édition électronique

URL : http://journals.openedition.org/communicationorganisation/2258

DOI : 10.4000/communicationorganisation.2258

ISSN : $1775-3546$

\section{Éditeur}

Presses universitaires de Bordeaux

Édition imprimée

Date de publication : 1 novembre 1999

ISSN : 1168-5549

Référence électronique

Violaine Appel, «Perrier : la mise en système médiatique d'une crise ", Communication et organisation [En ligne], 16 | 1999, mis en ligne le 27 mars 2012, consulté le 10 décembre 2020. URL : http:// journals.openedition.org/communicationorganisation/2258; DOI : https://doi.org/10.4000/ communicationorganisation.2258

Ce document a été généré automatiquement le 10 décembre 2020.

(c) Presses universitaires de Bordeaux 


\title{
Perrier : la mise en système médiatique d'une crise
}

\author{
Violaine Appel
}

1 Il n'est pas une seule entreprise, quelle que soit sa forme, qui puisse prétendre ne jamais avoir à affronter une rupture dans ses modalités habituelles de fonctionnement. Ces «crises", aujourd'hui conceptualisées et modélisées par nombre d'études sur les organisations, constituent probablement une des dimensions de la communication d'entreprise les plus ardues à maîtriser, tant les perturbations qu'elles provoquent placent souvent l'organisation en risque de «basculement d'image ». Le cas de la « crise Perrier » est à cet égard exemplaire.

2 Au lendemain de la seconde guerre mondiale, la petite Compagnie de la Source Perrier, «fournisseur breveté de Sa Majesté le roi d'Angleterre », passe aux mains de trois jeunes capitaines d'industrie : Jean Davray, Maurice Epry et Gustave Leven. Ce dernier deviendra le président directeur général du groupe et, pendant plus de quarante années, conduira son affaire jusqu'à en faire une société rayonnant dans cent vingt cinq pays. "Dans sa période faste des années quatre-vingt, Perrier détenait à elle seule $6 \%$ du marché américain des eaux minérales $»^{1}$.

3 Mais lorsqu'en février 1990, un taux de benzène supérieur à la norme est découvert dans certains échantillons, tous les efforts de Gustave Leven s'écroulent. L'information est largement diffusée par le système médiatique et l'image de pureté, de perfection et de «Champagne des eaux de table » de Perrier est alors remise en cause.

4 Très rapidement, le terme global de « crise » s'est imposé dans les médias pour recouvrir ce que l'on néglige trop souvent en évoquant ce cas : un nœud complexe d'interférences économiques, financières, identitaires et médiatiques. À partir de l'analyse comparative de documents écrits, cet article cherche à stigmatiser les procédures par lesquelles Perrier a tenté de contrôler cette complexité. Des contraintes pratiques nous ont imposé une limitation du corpus aux articles journalistiques parus dans une partie de la presse quotidienne nationale (France-Soir, La Croix, Les Échos, Le Figaro, L'Humanité, Libération, Le Monde) et dans deux revues spécialisées françaises (Communication CB News, Stratégie). La 
période d'étude couvre les quatre semaines qui ont suivi l'émergence de la crise, soit du 11 février au 9 mars 1990. L'ensemble des articles a d'abord fait l'objet d'une analyse de contenu sur le mode opératoire permettant l'étude objective, systématique et quantitative des thématiques développées et des procédés langagiers utilisés. Puis, nous avons cherché à interpréter, comprendre et analyser les données obtenues en les mettant en relation avec le contexte dans lequel s'inscrit la production de ces écrits. L'étude qui suit ne visant pas à rendre compte de l'étape descriptive, les citations d'extraits d'articles de presse seront donc peu nombreuses. Nous nous attacherons davantage à rendre compte les logiques d'intervention des différentes catégories d'acteurs impliquées.

5 À l'origine de l'affaire du benzène, il y a une erreur humaine liée aux procédures sanitaires : «le filtre chargé d'arrêter les impuretés contenues dans le gaz carbonique de la source n'a pas été changé dans les délais, laissant passer une quantité infinitésimale de benzène d'origine fossile dans l'eau $»^{2}$. Les doses de benzène relevées étant loin de représenter un danger pour la santé, cet incident n'aurait jamais du être porté à la connaissance des consommateurs. Il aurait suffit de changer le filtre en maintenant secrète l'information concernant cette erreur. Mais «la présence, parmi les employés, d'un «agent» au service de la concurrence donnera à celle-ci une toute autre importance. Muni de l'information, c'est lui qui déclenchera l'attaque $»^{3}$. Cet agent utilise l'incident initial, le relevé de taux de benzène anormal, et le déforme par un procédé d'exagération et de mélange vrai-faux suggérant un niveau de risque pour le consommateur sans rapport avec la réalité.

Il semble donc que l'on soit ici en présence d'une manifestation de ce qu'il est aujourd'hui convenu d'appeler la « guerre de l'information économique » dont Pascal Jacques-Gustave précise qu'elle «n'est pas une simple vue de l'esprit ou un concept à la mode. [...] Nulle entreprise n'est à l'abri d'une attaque informationnelle de ses produits ou d'un membre du personnel, de la découverte de substances toxiques dans des produits alimentaires, d'hypothétiques accidents dans la consommation de biens ou de services $»^{4}$. Et effectivement, le 12 février, Perrier retire 72 millions de bouteilles de la vente d'une valeur de 40 millions de dollars soit 225 millions de francs. Le 13, Suntory (importateur japonais) retire 10000 caisses du marché japonais. Le 14, la RFA stoppe les ventes de Perrier.

7 Depuis le 12 février, les ventes ont été suspendues aux États-Unis, au Canada, au Japon, en Allemagne, en Suisse, au Danemark et à Hongkong. Parallèlement, le 13 février, le déclenchement des ventes du titre à la bourse de Paris entraîne une suspension de la cotation. Cette situation rejaillit sur son principal actionnaire, Exor, dont le titre perd $7 \%$.

8 Toujours selon Pascal Jacques-Gustave, les actions de guerre d'informations économiques «ne prennent une importance destructrice que par la « mise en système médiatique » ». En d'autres termes, il s'agit de provoquer un événement ou un incident nuisible à l'entreprise cible et d'en assurer la publicité par les canaux médiatiques. Plus que l'incident, c'est sa médiatisation qui causera le plus de dommages. [...] « Dès lors qu'on utilise le relais médiatique, il n'existe plus de barrières géographiques. Plus que des " coups» limités à des négociations commerciales, les attaques informationnelles peuvent très rapidement prendre des proportions catastrophiques $»^{5}$. Le même auteur précise cependant que si l'information est « le principal outil de la guerre économique », elle sert «à la fois d'épée et de bouclier ». Et en effet, face aux proportions inquiétantes 
du développement de la crise, «l'entreprise doit réagir très vite et le fera en se battant sur le même terrain qui celui de l'attaque : l'information $»^{6}$.

9 L'entreprise Perrier met rapidement en œuvre une stratégie de nature défensive, basée sur la contre information. Celle-ci «peut être définie comme l'ensemble des actions de communication qui, grâce à une information pertinente et vérifiable, permettent d'atténuer, d'annuler [...] une attaque par l'information. La contre information [...] répond aux mêmes contraintes et requiert les mêmes qualités que l'attaque par l'information: renseignement préalable, maitrise des mécanismes psychologiques et psychosociologiques, maitrise des principes et techniques de communication (y compris publicitaires), connivence avec les médias de masse, etc. [...] Pour être crédible, la contre information s'attache à véhiculer de l'information ouverte et argumentée, non manipulée, donc facilement vérifiable. Elle est affaire de stratégie et de management de l'information $»^{7}$.

10 Durant toute la crise, on peut constater que l'espace rédactionnel consacré à la crise augmente proportionnellement à l'action de Perrier. Il est donc clair que l'entreprise a bien choisi le terrain de l'information comme base défensive. Mais, même si après vérification par un laboratoire, on peut affirmer que les traces de benzène décelées sont sans conséquence sur la santé du consommateur et même si après tests et contrôles l'étatmajor de Perrier peut affirmer haut et fort que la source est pure, il n'en reste pas moins que les quelques gouttes de benzène égarées dans les célèbres bulles risquent de mettre en péril l'image de l'entreprise et de ses produits.

11 Selon Éric Plottu « d'une façon générale, on peut considérer que le comportement d'une organisation, comme celui de tout système vivant, est commandé par la recherche de trois niveaux d'objectifs: un objectif de pérennité, de reproduction; un objectif de développement et un objectif d'optimisation du temps présent ». Il ajoute que «l'on peut associer, dans les organisations humaines, trois problématiques de choix (trois niveaux de décision) à ces trois types d'objectifs :

12 - les choix de rentabilité, d'optimisation du moment présent, qui relèvent d'une recherche de maximisation de la satisfaction au moindre coût (niveau opérationnel) ;

13 - les choix stratégiques définissant un mode et une trajectoire de développement de l'organisation (niveau stratégique) ;

14 - les choix identitaires relevant de l'objectif de pérennité et de reproduction de l'organisation (niveau identitaire) $»^{8}$.

15 Nous avons, dans ce qui suit, appliqué cette hiérarchisation au cas de la crise Perrier. La réponse de l'entreprise ne se limite pas à l'émission d'un volume suffisant d'informations. Elle se place aux trois niveaux de choix possibles.

La première résolution de la crise se place au niveau des choix opérationnels.

17 En effet, le non-respect des délais et donc des procédures lors du changement des filtres remet en cause des principes qui commandent les opérations de gestion courante, le comportement efficace des hommes, des normes de gestion, les critères de performance et de qualité fixés par les choix stratégiques.

Sauver l'image mais pas l'emploi !

19 Si les causes de cette crise sont à rapprocher d'un dysfonctionnement interne (recherche individuelle du moindre effort, non-adhésion aux orientations stratégiques, résistance au changement, sabotage, etc.), ni la Direction Générale du groupe Perrier, ni les médias 
n'ont été très loquaces sur le sujet. Les articles publiés dans la semaine qui suit l'événement ne font mention d'aucune information sur la manière dont Perrier a géré la crise à l'intérieur de son entreprise. Or, cet incident a eu des répercussions au sein de celle-ci : à court terme, l'ingénieur responsable du nettoyage des filtres a été licencié ; à moyen et long termes l'usine de Vergèze a perdu $1 / 3$ de ses effectifs en 5 ans. Les déboires de Perrier ont donc été un drame pour cette région où le taux de chômage dépassait $14 \%$ en 1994. Dans cette petite commune, une personne sur trois travaillait chez Perrier.

Il semblerait donc que la résolution de cette crise interne ne soit pas l'aboutissement d'un dialogue ou d'une négociation au sein de l'organisation mais résulte exclusivement des intérêts enjeu dans la confrontation de deux logiques d'acteurs: l'entreprise dans une logique marchande (et non dans une logique de heu social) et les médias dans une logique commerciale et informationnelle.

21 En effet, ce choix renvoie probablement à la nécessité d'avoir, dans un premier temps, une action de type factuel qui doit prendre en compte l'émotion suscitée par les conséquences éventuelles et «l'angoisse de l'opinion qui ne peut comprendre les notions techniques très développées. [...] L'information gagnera à être simplifiée le plus possible, tout en restant vraie, pour pouvoir être comprise par le plus grand nombre ${ }^{9}$. 
30 - une prise de décision rapide, limitant ainsi la possibilité d'un processus délibératif dans la prise de décision pour privilégier l'application d'une procédure d'urgence prédéterminée ;

31 - une décision significative, c'est-à-dire une décision impliquant la mise en œuvre d'actions significatives aptes à résoudre l'état d'urgence $»^{13}$.

32 Dans le cas Perrier, le groupe réagit vite en jouant la carte de la transparence pour préserver son image. Ainsi, Gustave Leven, l'un des patrons français les plus réservés, décide de prendre la parole et d'ouvrir les portes de l'usine du Gard aux journalistes qui le souhaitent. Pourtant, cette usine d'embouteillage de Perrier, siège officiel de l'empire, passait depuis longtemps pour une sorte de "cité interdite ». Les photographes étaient interdits de séjour, les journalistes refoulés et adressés à la direction parisienne.

33 Cette tradition du silence est soudainement brisée. Perrier a fait le « vide " pour créer des conditions d'écoute : vide dans les rayons, vide par rapport à une attitude antérieure de " protectionnisme ». Le choix d'un seul porte-parole permet, d'une part, de préparer une ligne de communication unique de réponses ou d'initiatives, et, d'autre part, de limiter la part de risque liée à la divulgation de toute information. En effet « chaque prise de parole est une opportunité d'action au service de la stratégie. Bien utilisée, elle permet de conforter ses positions ${ }^{14}$. Le choix de la transparence doit permettre d'éviter de créer artificiellement un nouveau scoop qui ferait redémarrer la crise.

34 Car pour être crédible il faut dire la vérité. Or « en matière de communication, la difficulté consiste à comprendre la perception de la vérité de l'autre, à convenir d'une perception commune $»^{15}$. Si la première attitude spontanée est souvent déterminée par les sentiments, ce sont les explications et le raisonnement qui modifient, par la suite, les comportements. «Le rationnel corrige l'irrationnel, l'explication émousse l'émotion, le raisonnement forge le sens et la compréhension $»^{16}$.

Pour Perrier, il ne faut donc pas aller contre des réactions irrationnelles du public ou des médias mais il s'agit de comprendre l'univers irrationnel des acteurs pour savoir sur quels fondements appuyer la communication. Car la stratégie consiste aussi à penser avec l'esprit de «l'adversaire». Ainsi, la recherche d'une cause identifiable, lisible et acceptable de la crise, la sanction prise par Perrier à l'égard de ce que l'on pourrait appeler un bouc émissaire, la possibilité offerte aux journalistes de s'approprier la situation nouvelle, de pouvoir l'appréhender et l'intégrer à leur logique médiatique, sont autant d'éléments qui permettent de désamorcer la crise, qui aident à l'accepter. La part d'inconnu révélée à l'occasion de la crise est alors, sinon supprimée, tout du moins estompée.

L'annonce, par Gustave Leven, du retrait de 160 millions de bouteilles des marchés du monde entier, a en fait joué un double rôle :

37 - anticiper la réaction du consommateur et transformer ce qui aurait pu être un grave revers pour son groupe en une « presque victoire ». La direction de Perrier a considéré le public non pas seulement comme un client mais comme un premier rôle dans le scénario de crise. Il est devenu un acteur de cette mise en scène,

38 - permettre un gros « coup » médiatique en faisant des médias les porte-parole de toute la publicité de la firme pendant cette période..

39 Mais, la prise de décision dans l'urgence est incompatible avec le temps nécessaire aux décisions relevant du stratégique. Il convient donc de prendre des décisions rapides qui 
ne remettent pas fondamentalement en cause ni les orientations stratégiques, ni l'identité mais qui s'inscrivent dans une même logique : récréer de la stabilité, gérer le changement dans la continuité des logiques définies à long terme.

«Bien que reposant sur des logiques et temporalités opposées, la mise en œuvre synchronique des deux processus de décision peut ainsi permettre à l'organisation de surmonter la situation de crise, le temps court de la décision répondant à l'état d'urgence « ménageant le temps » pour le processus délibératif de résolution de la crise $»^{17}$. Il s'agit de reconstruire un nouvel équilibre, de faire renaître du sens.

C'est pour cette raison que les choix identitaires de l'entreprise sont cruciaux en situation de crise. L'affaire du benzène a ébranlé l'identité même de l'organisation, ses valeurs fondamentales, sa mission et son environnement. L'identité de l'entreprise et du produit sont profondément affectées : on constate que l'organisation, face à un environnement turbulent, est momentanément dépossédée de son univers de référence et de ses points de repères, ceux qui donnent du sens aux actions de l'entreprise.

Durant tout le mois de février 1990, l'affaire Perrier a inspiré les journalistes qui ont su décliner des titres jouant avec le fameux slogan « Perrier, c'est fou » : «Perrier : au sec !", «Perrier : revers gagnant », « Perrier, l'eau qui fait plouf !», etc.

À la lecture de ceux-ci on s'aperçoit qu'une confusion permanente est entretenue entre le nom du produit et celui de l'entreprise. Cette ambiguiité, cultivée depuis toujours par l'entreprise, permet d'atteindre un double objectif, en associant communication produit et communication institutionnelle.

Une telle logique d'emboîtement va, lors de cette crise, permettre à Perrier de redonner du sens à son produit et à sa raison d'être en tant qu'entreprise..

45 Tout d'abord, le retour de Perrier dans les rayons se fait essentiellement sur la scène médiatique, la même qui avait nuit à son image. L'entreprise réussit alors à réinvestir positivement et à maitriser un terrain miné, une sorte de cacophonie médiatique.

En effet, moins d'un mois après l'annonce de traces de benzène, une campagne presse paraît dans l'ensemble de la presse régionale et nationale pour annoncer le plan de lancement international de la même bouteille verte avec une nouvelle étiquette portant la mention «nouvelle production ».

47 L'effet de surprise créé lors de ce retour rapide se fait en quelque sorte l'écho de celui créé par « l'effet benzène ».

Par ailleurs, cette annonce presse, liée directement au produit, s'accompagne d'une campagne télévisuelle française qui, tout en parlant produit, distille habillement un message qui s'adresse à la société et à la culture française et qui permet de redonner du sens à l'entreprise et à son activité industrielle. "À la manière de la célèbre « Femme du boulanger » de Pagnol, une voix ressemblant à celle de Raimu murmure : «je savais que tu reviendrais que tu pouvais pas me faire ça, c'était pas ton genre, «fiérote » comme tu es de tout laisser tomber, et que tu as été tout casser... là... Allez viens petite, avec ta belle étiquette, tu es toute pure là où y'a marqué Nouvelle Production... que ça te va bien tu sais... et que tu vas me l'abîmer si tu continues à pleurer comme ça... ». La bouteille qui versait alors une larme, répond avec une voix de femme: "Je ne pleure pas, c'est le bonheur ». "Té, tu crois que je ne le sais pas » conclut le nouveau Raimu » ${ }^{18}$. On joue ici de référents culturels presque emblématiques, associant habilement authenticité et simplicité. S'écartant ainsi du discours pseudo-scientifique qui aurait pu combiner batailles de chiffres et d'expertises, Perrier réintroduit un rapport de connivence 
psychologique et physique (géographique) avec le consommateur français. Un retour aux racines, un réancrage dans certaines valeurs traditionnelles fondamentales : il n'en fallait pas plus pour repositionner la petite bouteille verte.

Mais ce repositionnement identitaire ne doit cependant pas faire oublier deux conséquences importantes de cette crise dans un parcours jusqu'alors sans faute.

Le retrait des produits de la vente et la vaste campagne médiatique de leur retour sur le marché ont eu un coût financier très élevé. Sans compter la versatilité des consommateurs qui se sont tournés vers d'autres produits concurrents.

51 Par ailleurs, des pertes d'emplois vont venir s'ajouter aux pertes financières. En effet, si dans un premier temps l'entreprise a embauché une main d'œuvre saisonnière pour pouvoir rapidement reconstituer le stock, deux vagues de licenciements vont réduire d'un tiers le nombre des effectifs de l'usine de Vergèze. Sur 3000 employés que comptait l'usine, 450 seront licenciés en 1992 et 600 en 1995. Cette crise économique et sociale de toute une région a cependant fait couler beaucoup moins d'encre...

De prime abord, la crise du benzène pourrait s'appréhender suivant un modèle stimulus/ réponse classique: à la mise en système médiatique d'une attaque informationnelle, certes classique, mais aux conséquences dramatiques, Perrier a opposé avec succès une mise en scène médiatique d'une campagne associant publicité et communication institutionnelle. Mais, au-delà de ce qui est donné à voir, la réponse de l'entreprise a sollicité tous les champs d'actions possibles. Par la combinaison des niveaux opérationnel, stratégique et identitaire, Perrier a cumulé les traitements de la crise à court, moyen et long termes, négligeant parfois ce qui aujourd'hui ne peut plus l'être : sa responsabilité sociale, son engagement « citoyen».

Il reste que l'étude présentée ici ne prend pas en compte la dimension internationale de la crise qui n'a pu qu'influer sur les choix opérés au niveau identitaire. Toutefois, un repli ou un réancrage dans ses territoires originels constituent les premières étapes indispensables à un redéploiement de l'activité et justifient ainsi l'intérêt que l'on peut leur porter. Car l'affaire du benzène a beau être l'un des exemples de crise les plus fréquemment évoqués, son ampleur et son niveau de complexité la rendent difficile à déchiffrer et à expliquer.

Les attaques informationnelles sont aujourd'hui au cœur des enjeux commerciaux d'entreprises présentes sur des marchés peu évolutifs en parts de marchés, tels que l'industrie aéronautique, l'informatique, l'automobile, l'eau, etc. La procédure de retrait global des produits, inaugurée par Perrier, est actuellement fréquemment reproduite par des entreprises placées dans des situations comparables. Dans ces conditions, il conviendrait de s'interroger sur la pertinence de l'usage de techniques particulières de communication de crise alors qu'elles semblent aujourd'hui devoir s'inscrire de façon permanente dans une stratégie de communication globale. 


\section{BIBLIOGRAPHIE}

\section{BIBLIOGRAPHIE}

COMMUNiCATION CB NEWS, « RSCG fait craquer Perrier », in CB News, n 160,12 mars 1990.

DENNERY Michel, « Les crises ça n'arrive pas qu'aux autres », I.N.E. - C.E.T.I.E. - Congrès de Bruges, 25 avril 1997.

GHANEM-DOMONT Cynthia, « Saga Perrier : de la folie à l'ivresse », in Supplément Sagas Stratégies, $\mathrm{n}$

- 1061 du 19 juin 1998, p. 86.

HARBULOT Christian, JACQUES-GUSTAVE Pascal, « Manœuvre médiatique et compétition

économique », in Enjeux Atlantiques, 1998.

JACQUES-GUSTAVE Pascal, « Guerre et contre-guerre de l'information économique », in Échanges, 1994.

LOMBARD Marie-Amélie, « Perrier : du charivari dans les bulles », in Le Figaro, 15 février 1990.

PERNICAUT Nicole, « Le coup de Perrier remonte le titre », in Libération, 16 février 1990.

PLOTTU Éric, «Les crises et leur gestion », in Sciences de la Société : Urgence et décision, Presses Universitaires du Mirail, n 44, mai 1998, pp. 145-163.

\section{NOTES}

1. Ghanem-Domont Cynthia, "Saga Perrier : de la folie a l'ivresse ", in Supplément Sagas Stratégies, $n^{\circ} 1061$ du 19/6/1998, p. 86.

2. Ghanem-Domont Cynthia, «Saga Perrier : de la folie à l'ivresse », op. cit., p. 86.

3. Ghanem-Domont Cynthia, « Saga Perrier : de la folie à l'ivresse », op. cit., p. 86.

4. Jacques-Gustave Pascal, « Guerre et contre-guerre de l'information économique », in Échanges, 1994

5. Jacques-Gustave Pascal, « Guerre et contre-guerre de l'information économique », op.cit.

6. Jacques-Gustave Pascal, « Guerre et contre-guerre de l'information économique », op.cit.

7. Harbulot Christian, Jacques-Gustave Pascal, «Manœuvre médiatique et compétition économique ", in Enjeux Atlantiques, 1998.

8. Plottu Éric, "Les crises et leur gestion », in Sciences de la Société: Urgence et décision, Presses Universitaires du Mirail, n 44, mai 1998, p. 147.

9. Dennery Michel, «Les crises ça n'arrive pas qu'aux autres », IN.E.. - C.E.T.I.E. - Congrès de Bruges, 25 avril 1997.

10. Pernicaut Nicole, «Le coup de Perrier remonte le titre ", in Libération, 16 février 1990.

11. Lombard Marie-Amélie, « Perrier : du charivari dans les bulles », in Le Figaro, 15 février 1990.

12. Lombard Marie-Amélie, «Perrier : du charivari dans les bulles», op. cit.

13. Plottu Éric, « Les crises et leur gestion », op.cit., p. 155.

14. Dennery Michel, «Les crises ça n'arrive pas qu'aux autres », op.cit.

15. Dennery Michel, «Les crises ça n'arrive pas qu'aux autres », op.cit. 
16. Dennery Michel, «Les crises ça n'arrive pas qu'aux autres », op.cit.

17. Plottu Éric, « Les crises et leur gestion », op.cit., p. 156.

18. Communication CB News, « RSCG fait craquer Perrier », in Communication CB News, $\mathrm{n}^{\circ} 160,12$ mars 1990.

\section{RÉSUMÉS}

La crise Perrier a fait couler beaucoup d'encre dans les médias mais l'absence d'analyse du phénomène de mise en système médiatique en tant que tel nous a amenée à constituer cette crise en objet d'étude. À travers les logiques d'interventions des différentes catégories d'acteurs impliquées, nous avons cherché à stigmatiser les procédures par lesquelles Perrier a tenté de contrôler la complexité de l'événement, en opposant à une attaque informationnelle bien orchestrée, une stratégie combinant plusieurs problématiques de choix.

The Perrier crisis has provoked a lot of comments in the media but the lack of analysis of the new media staging as involved by the phenomenon has led us to consider it as a case study. Through the ways the different categories of actors have been implied, we have tried to point out the procedures used by Perrier to control such a complex event: opposing a strategy combining several outstanding choices to a well-organised media attack.

\section{AUTEUR}

\section{VIOLAINE APPEL}

Violaine APPEL est maître de conférences en sciences de l'information et de la communication à l'Université Nancy 2. Depuis deux ans, elle dirige l'option « communication d'entreprise » au département Information-Communication de l'IUT. Ses travaux de recherche portent essentiellement sur les problématiques de communication interne et externe des entreprises et des organisations. 